\title{
EQUUS (AMERHIPPUS) NEOGAEUS LUND, 1840 (PERISSODACTYLA, EQUIDAE) NO QUATERNÁRIO DO ESTADO DO PARANÁ, BRASIL
}

\author{
DAVID DIAS DA SILVA \\ Programa de Pós-Graduação em Geociências, Instituto de Geociências, UFRGS, Campus do Vale, \\ 91501-970, Cx.P. 15001, Porto Alegre, RS, Brasil.david.paleo@hotmail.com \\ FERNANDO ANTONIO SEDOR \\ Museu de Ciências Naturais, Universidade Federal do Paraná, Rua Cel. Franscisco Heráclito dos Santos, 100, \\ Curitiba, PR, Brasil.sedor@ufpr.br \\ ANA MARIA RIBEIRO \\ Museu de Ciências Naturais, FZB/RS, Av. Dr. Salvador França, 1427, 90690-000, \\ Porto Alegre, RS, Brasil. ana.ribeiro@,fzb.rs.gov.br
}

\begin{abstract}
EQUUS (AMERHIPPUS) NEOGAEUS LUND, 1840 (PERISSODACTYLA, EQUIDAE) FROM THE QUATERNARY OF PARANÁ STATE, BRAZIL. The fossil record of Equidae in southern Brazil is restricted to Rio Grande do Sul State and is represented by isolated teeth and fragmentary cranial and postcranial remains. This paper describes Equus (Amerhippus) neogaeus Lund from Paraná State, where Pleistocene mammals are poorly known. The material is a left dentary with cheek teeth and was found in the Mangueirinha Municipality in a small alluvial terrace, associated with some remains of Gomphoteriidae and Megatheriidae. The presence of E. (A.) neogaeus suggests an open paleoenvironment with xerofitic vegetation, and could indicate a Lujan Age/Stage (late Pleistocene/early Holocene) for the assemblage fauna.
\end{abstract}

Key words: Quaternary, megafauna, south of Brazil, holartic ungulate.

RESUMO - O registro fóssil de Equidae para a região sul do Brasil está restrito ao Estado do Rio Grande do Sul, representado por material fragmentário e dentes isolados. Este trabalho descreve Equus (Amerhippus) neogaeus Lund para o Quaternário do Estado do Paraná, onde os mamíferos pleistocênicos são pobremente conhecidos. O material está representado por um dentário esquerdo incompleto com toda a série molariforme, procedente do Município de Mangueirinha, e foi encontrado em um pequeno terraço aluvionar associado a alguns ossos e dentes de Gomphotheriidae e Megatheriidae. E. (A.) neogaeus sugere paleoambiente aberto com vegetação xerofítica, sendo que sua presença poderia indicar uma Idade/Piso Lujanense (Pleistoceno superior-Holoceno inferior) para a assembleia.

Palavras-chave: Quaternário, megafauna, sul do Brasil, ungulado holártico.

\section{INTRODUÇÃO}

Os equídeos chegaram à América do Sul posteriormente ao soerguimento do Istmo do Panamá durante o evento do Grande Intercambio Biótico Americano (GIBA) (Webb, 1991). Comumente, os equídeos fósseis sul-americanos são distribuídos em dois grupos: "hipidiformes" e "equidiformes", sendo que ambos os grupos apresentam formas de pequeno e grande porte, provavelmente por colonizarem ambientes semelhantes (MacFadden, 1997; Alberdi \& Prado, 2004). Durante o GIBA, os Equidae, a exemplo dos Gomphotheriidae, distribuíram-se preferencialmente através de duas grandes rotas que definiram distintos padrões de ocorrência dos imigrantes holárticos (Webb, 1991). As formas pequenas de equídeos utilizaram o corredor andino para sua dispersão, enquanto que as formas de grande porte distribuíram-se através da rota oriental e de algumas regiões costeiras (Alberdi \& Prado, 1992; Sanchéz et al., 2006).
A ocorrência mais antiga de Equidae para a América do Sul corresponde a Hippidion Owen, 1869, procedente da localidade de Uquía, norte da Argentina, de uma camada atribuída ao Plioceno superior-Pleistoceno inferior, datada em torno de 2.5 Ma (Marshall et al., 1982; Prado et al., 1998; Reguero et al., 2007). Para a América do Sul atualmente são aceitas três espécies de hipidiformes: Hippidion principale (Lund, 1845), Hippidion devillei (Gervais, 1855) e Hippidion saldiasi (Roth, 1899). Hippidion é registrado em afloramentos pleistocênicos/holocênicos na Colômbia, Equador, Peru, Bolívia, Paraguai, Chile, Argentina, Uruguai e Brasil (Alberdi \& Prado, 1992, 1993, 1995, 2004; Carlini \& Tonni, 2000).

O gênero Equus Linnaeus, 1758 tem a sua primeira ocorrência na localidade de Tarija, Bolívia, (Alberdi \& Prado, 1992; Alberdi \& Prado, 1995) atribuído por MacFadden et al. (1983) ao Pleistoceno médio (1.0-0.7 Ma AP). De acordo com Alberdi \& Prado (1995) são reconhecidas cinco espécies para o continente sul-americano: Equus (Amerhippus) andium 
Branco, 1883; Equus (Amerhippus) insulatus Ameghino, 1904; Equus (Amerhippus) lasallei Daniel, 1948; Equus (Amerhippus) santaeelenae (Spilmann, 1938) e Equus (Amerhippus) neogaeus Lund, 1840.

O registro da distribuição de Equus durante o Quaternário compreende a Venezuela, Colômbia, Equador, Peru, Chile, Bolívia, Paraguai, Argentina, Uruguai e Brasil (Alberdi \& Prado, 1992, 1995; 2004; Prado \& Alberdi, 1994; Alberdi \& Frassinetti, 2000; Carlini \& Tonni, 2000; Martinez, 2004; Salas et al., 2004; Rincón et al., 2006). Ambos os gêneros são extintos no limite Pleistoceno-Holoceno há cerca de 10.000 anos AP (Ficcarelli et al. 2003).

No Brasil os primeiros estudos sobre cavalos fósseis foram realizados pelo naturalista dinamarquês Peter W. Lund. O material coletado e estudado por Lund (1840, 1842, 1846), procedente das cavernas calcárias da região de Lagoa Santa em Minas Gerais, foi enviado à Dinamarca e posteriormente estudado por Boas (1881) e Winge (1906). Durante o Pleistoceno os Equidae no Brasil estavam representados pelos gêneros Equus (Amerhippus) e Hippidion procedentes principalmente da região Nordeste, Sudeste e Sul do país. O gênero Hippidion é registrado para os estados de Ceará, Piauí, Rio Grande do Norte, Paraíba, Alagoas, Bahia, Minas Gerais e Rio Grande do Sul, e Equus (Amerhippus) para Piauí, Ceará, Rio Grande do Norte, Paraíba, Pernambuco, Alagoas, Bahia, Minas Gerais e Mato Grosso do Sul (Lund, 1840, 1842, 1846; Price, 1944; Vidal, 1946; Souza Cunha, 1959, 1981; Rolim, 1971; Bergqvist et al., 1987; Cartelle et al., 1989; Cartelle, 1992; Oliveira, 1992; Guérin, 1991; Alberdi et al., 2003;
Porpino et al. 2004; Salles et al. 2006; Dias Neto et al., 2008; Silva et al. 2010; Ghilardi et al. 2011) (Figura 1).

$O$ registro de equídeos quaternários procedentes da região sul do Brasil se restringem principalmente ao Estado do Rio Grande do Sul (RS) e consiste praticamente de dentes e ossos isolados em estado fragmentário (Paula Couto, 1944; SouzaCunha, 1950; 1959; Kerber \& Oliveira, 2008a,b; Ribeiro \& Scherer, 2009). No Estado do Paraná, há apenas uma citação de Ribeiro et al. (2005) para o Município de Mangueirinha. Portanto, o presente trabalho tem como principal objetivo descrever material de Equidae para o Pleistoceno do Estado do Paraná, bem como, com base na paleofauna encontrada associada, fazer algumas inferências paleoambientais.

\section{MATERIAL E MÉTODOS}

O material estudado consiste de um dentário esquerdo incompleto com toda a série molariforme preservada e encontra-se depositado na coleção de Paleontologia do Museu de Ciências Naturais do Setor de Ciências Biológicas da Universidade Federal do Paraná. As medidas foram realizadas com um paquímetro com precisão de $0,01 \mathrm{~mm}$ e expressas em milímetros. A nomenclatura anatômica e os parâmetros métricos foram baseadas em Eiseinmann et al. (1988). A sistemática utilizada no presente trabalho está de acordo com os trabalhos de Alberdi \& Prado (1992, 1995, 2004).

Abreviaturas institucionais. COPEL, Companhia Paranaense de Energia Elétrica; MCL, Museu de Ciências Naturais da Pontifícia Universidade Católica de Minas Gerais,

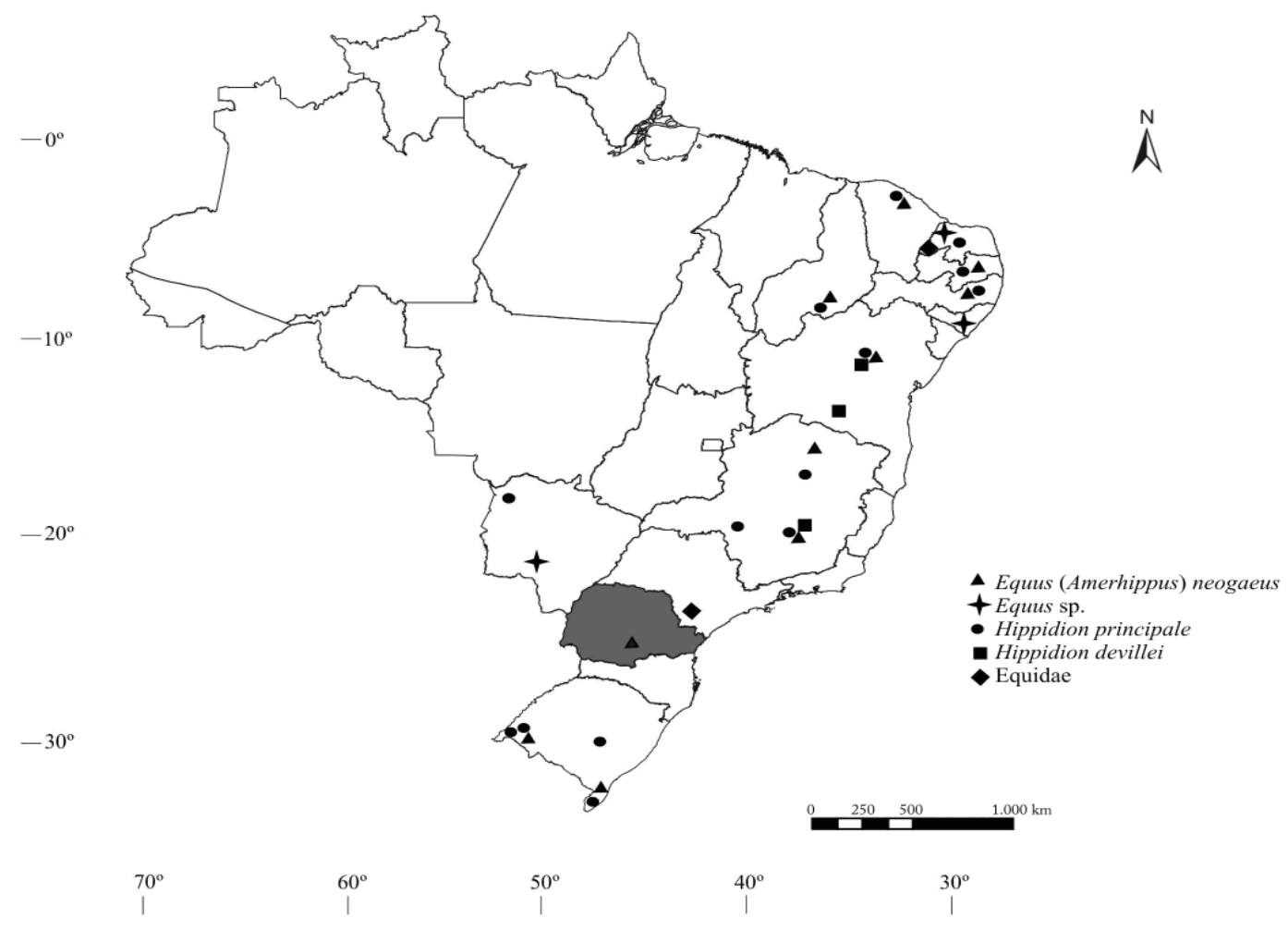

Figura 1. Mapa da distribuição da ocorrência de Equidae para o Pleistoceno do Brasil. Referências no texto.

Figure 1. Distribution map of the occurrence of Equidae for Pleistocene of Brazil. References in the text. 
Belo Horizonte, MG; MCN.P, Museu de Ciências Naturais da Universidade Federal do Paraná, Curitiba, PR; MCPUPV, Museu de Ciências da Pontifícia Universidade Católica de Uruguaiana, RS; SGO.PV, Museo Nacional de Historia Natural de Santiago de Chile.

\section{LOCALIZAÇAO GEOGRÁFICA E ASPECTOS GEOLÓGICOS DO JAZIGO}

O espécime foi coletado em fevereiro de $1991 \mathrm{em}$ um pequeno depósito sedimentar, localizado em um terraço aluvionar de superfície plana e situado a $50 \mathrm{~m}$ da margem esquerda do Rio da Divisa, afluente do Rio Iguaçu, no Município de Mangueirinha, Estado do Paraná (254ㄱ'30”S, $\left.52^{\circ} 07^{\prime} 00^{\prime} \mathrm{O}\right)$ (Figura 2). Atualmente esta localidade está inacessível, pois se encontra encoberta pelas águas da represa da Usina Hidrelétrica Governador Ney Aminthas de Barros Braga da COPEL.

O sítio localiza-se em uma área que foi tectonicamente muito movimentada, situada sobre a Falha da Divisa. A sua litologia constitui-se de argilas e conglomerados cobertos por um nível superficial areno-argiloso com espessura média de 2,5 m, assentado sobre um substrato de basalto maciço, cinza escuro e subafanítico da Formação Serra Geral da bacia do Paraná ( 120-130 Ma). O conglomerado é muito resistente e nele predominam seixos de basalto maciço em uma proporção de até $75 \%$, envolvidos em uma matriz limonitizada, também ocorrem seixos de quartzo e basalto amigdaloidal. Os clastos não apresentam qualquer padrão ou direção preferencial, distribuição ou estratificação. São desde angulosos e facetados até arredondados com alto grau de esfericidade, evidenciando transporte com retrabalhamento. A matriz tem constituição areno-síltica argilosa, com fragmentos de sílex, quartzo, ágata, opala e raras palhetas milimétricas de moscovita e provavelmente pirita. A limonitização ocorreu em diferentes graus, atingindo coloração preta, com brilho vítreo e iridescência.

Na secção B-B' (Figura 3) observa-se o contato brusco e subvertical com o conglomerado, e também como preenchimento, em forma de bolsão, ocorre uma faixa de aproximadamente $2 \mathrm{~m}$ de espessura de argila plástica homogênea de coloração cinza média a escura, com grânulos esparsos e caoticamente distribuídos. Esta argila transiciona lateralmente para material mais síltico, cinza claro a médio, com alguns níveis de areia fina de coloração amarelada, onde foi encontrado o espécime aqui estudado. O material

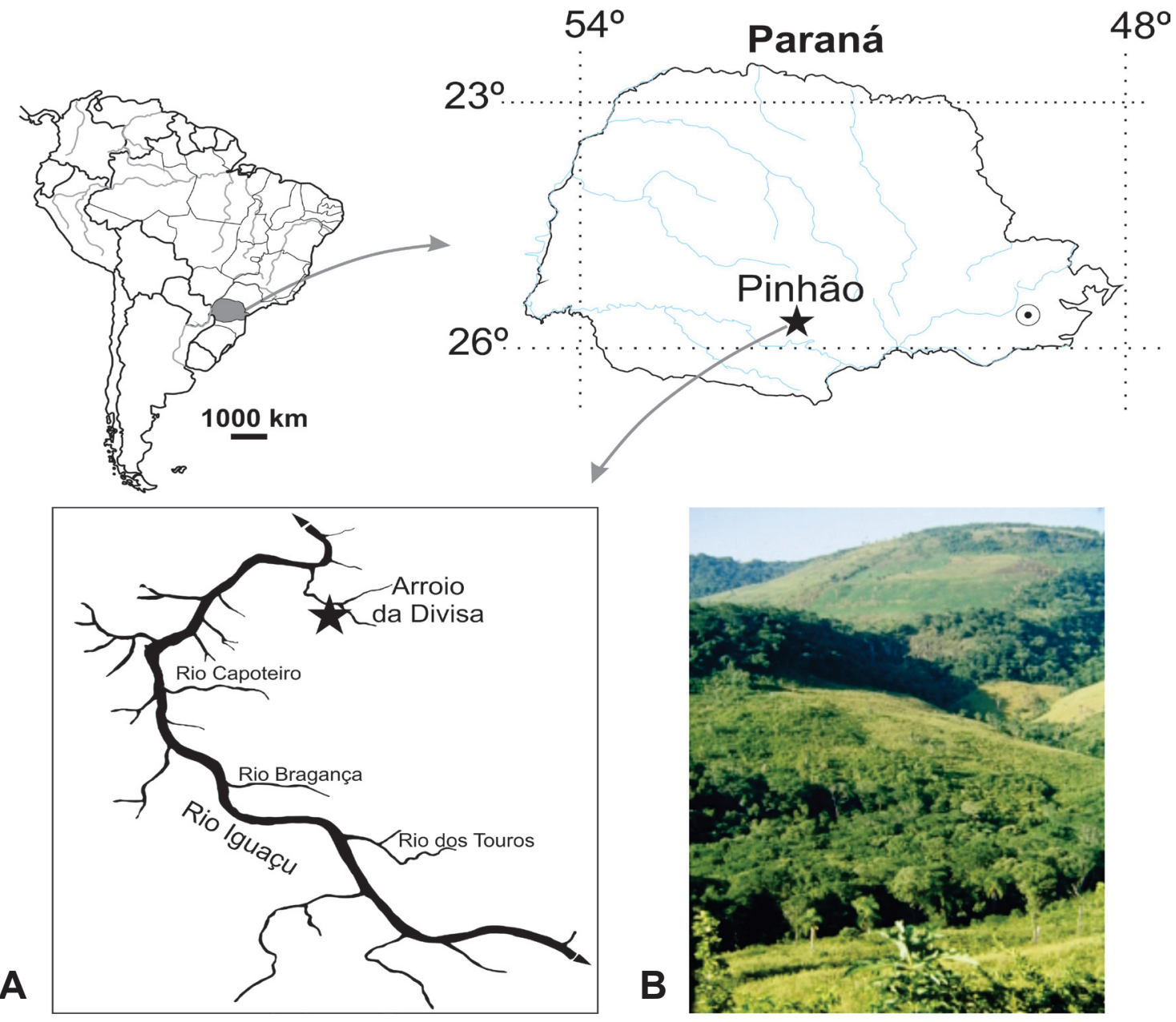

Figura 2. A, mapa da localização do sítio fossilífero; B, foto da área do sítio fossilífero.

Figure 2. A, location map of the outcrop; B, photo of the outcrop area. 

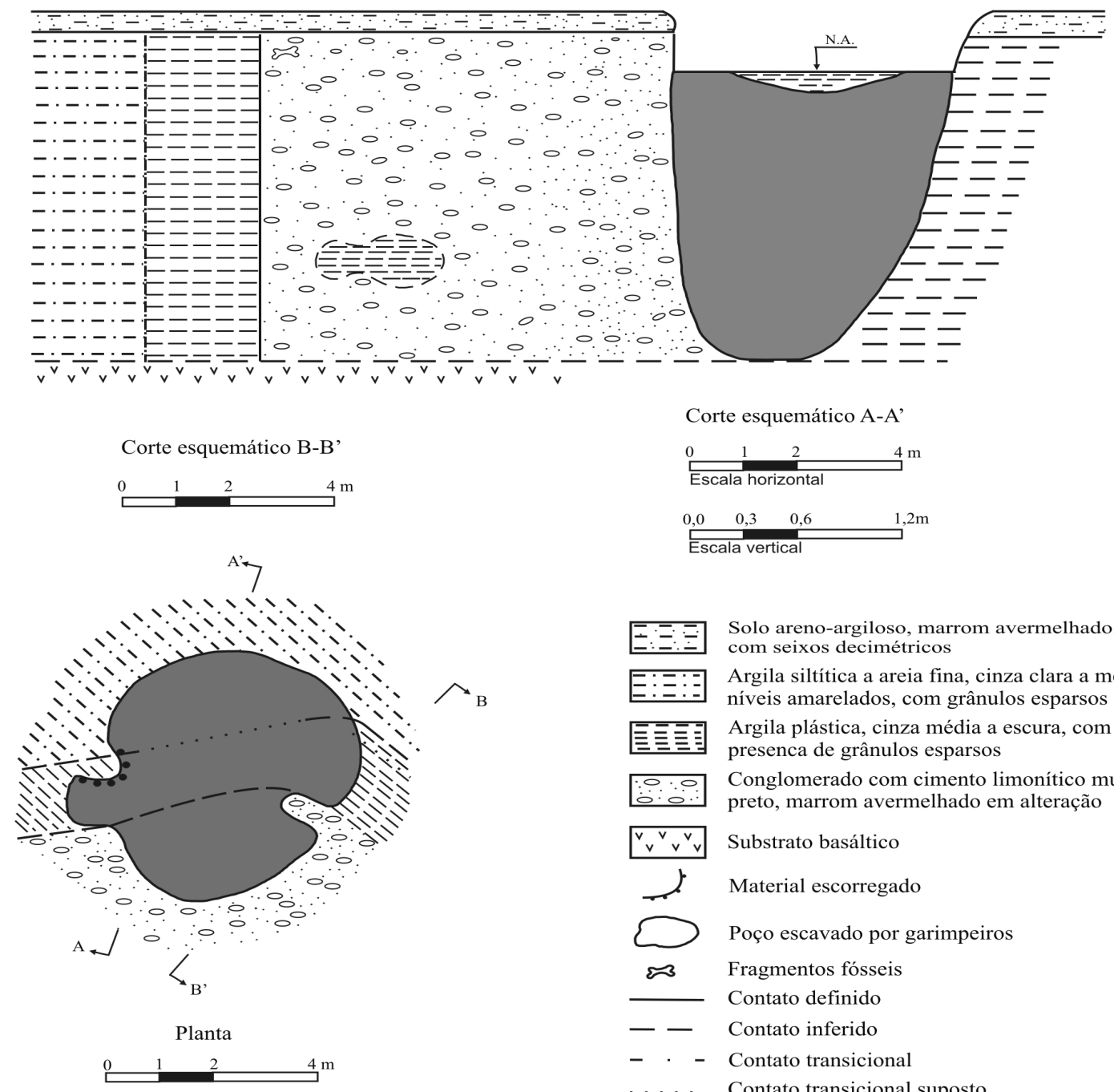

Corte esquemático A-A'

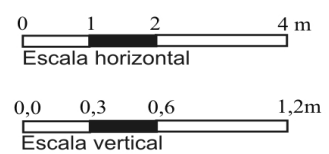

- $=$ Solo areno-argiloso, marrom avermelhado, friável,

com seixos decimétricos

E: : $:$ Argila siltítica a areia fina, cinza clara a média,

níveis amarelados, com grânulos esparsos

E互国 Argila plástica, cinza média a escura, com presenca de grânulos esparsos

0.0 Conglomerado com cimento limonítico muito resistente,

preto, marrom avermelhado em alteração

${ }^{v} v^{\vee} v^{v} v$ Substrato basáltico

Material escorregado

$\bigcirc$ Poço escavado por garimpeiros

$\approx \quad$ Fragmentos fósseis

Contato definido

- Contato inferido

- - Contato transicional

.... Contato transicional suposto

Figura 3. Perfil esquemático do sítio fossilífero.

Figure 3. Schematic profile of outcrop.

argiloso está recoberto por uma capa aluvio-coluvionar de aproximadamente $0,2 \mathrm{~m}$.

A gênese deste pequeno depósito pode estar relacionada a eventos tectônicos, pois sua localização esta associada a uma falha. Provavelmente os ossos dispersos ao longo da linha de drenagem tenham sido arrastados pelas enxurradas e reunidos nesta pequena depressão juntamente com os sedimentos grossos, possivelmente durante alguns eventos pluviométricos episódicos.

Todo o preenchimento sedimentar deste pequeno depósito foi metodicamente removido e peneirado. Este procedimento revelou uma assembleia fóssil que além do espécime aqui estudado (Equidae), reúne dentes e ossos pós-cranianos atribuídos a Megatheriidae e Gomphoteriidae, além de outros fragmentos ósseos não determinados.

Os ossos apresentam feições tafonômicas que evidenciam graus variáveis de exposição aérea e rolamento. Estas feições, bem como a natureza do material conglomerático, denotam pouco transporte e retrabalhamento (Figura 3).
Os vários níveis de oxidação diferencial dos horizontes conglomeráticos provavelmente são consequentes de variações de nível freático, que proporcionaram uma intensa cimentação, criando uma superfície impermeável e resistente que possibilitou a preservação destes ossos.

\section{SISTEMÁTICA PALEONTOLÓGICA \\ Ordem PERISSODACTYLA Owen, 1948 Família EQUIDAE Gray, 1921}

Equus Linnaeus, 1758

Equus (Amerhippus) Hoffstetter, 1950

Espécie tipo. Equus (Amerhippus) andium Branco, 1883, ex Wagner, 1860.

Equus (Amerhippus) neogaeus Lund, 1840

(Figuras 4A-C) 
Material. MCN.P. 504, dentário esquerdo com toda a série molariforme.

Descrição. Dentário: O dentário está fragmentado anteriormente na porção do diastema e falta os processos angular, condilar e coronoide, medindo $320 \mathrm{~mm}$ de comprimento até a borda posterior do ramo ascendente e $93,7 \mathrm{~mm}$ de altura. Toda a borda ventral da região média do dentário está faltando, expondo os alvéolos dentários e observam-se as raízes já fechadas apenas do pm2 ao pm4 (Figuras 4A-B). Abaixo do $\mathrm{pm} 3$, ocorre uma fratura de formato semicircular com 7,09 $\mathrm{mm}$ de diâmetro. O ramo ascendente é pouco espesso médiolateralmente, sendo mais notável posteriormente e abaixo do forame mandibular. A região dorsal posterior ao m3 está faltante, podendo observar, em vista dorsal, a região interna do forame mandibular (Figura 4C).

Dentição: O comprimento total da série dentária é de 163,00 mm (Figura 4C). A altura de toda série vai diminuindo no sentido mesiodistal. Está faltando a região labial da porção apical do linguafléxido, metastilido e metacônido do pm4. Os pré-molares e molares apresentam metacônido arredondado e metastilido angular, completamente unidos formando um "laço duplo". As bordas labiais do protocônido e do entocônido são relativamente retilíneas em toda a série dentária. $\mathrm{O}$ linguafléxido é bastante raso no pm2, e no restante dos pré-molares e molares apresenta formato arredondado, achatado e angular, em forma de "V". O ectofléxido é raso nos pré-molares e profundo nos molares penetrando o istmo, porém nunca alcança o linguafléxido. Apresenta prega cavalina, presente em toda a série molariforme, sendo mais visível no pm2 e pm3. As medidas (mm) dos diâmetros mesiodistal e bucolingual dos pré-molares estão apresentados na Tabela 1.

\section{DISCUSSÃO E CONCLUSÕES}

A determinação do espécime foi realizada de acordo com as características diagnósticas apresentadas por Alberdi \& Prado $(1992,1993,1995)$ para o gênero Equus e com base na comparação com o material depositado na Pontifícia Universidade Católica do Rio Grande do Sul, Uruguaiana e dos espécimes figurados por Souza-Cunha (1981), Ubilla \& Alberdi (1990), Scherer \& Da Rosa (2003), Alberdi et al. (2003), Rincón et al. (2006) e Prado \& Alberdi (2008).

O espécime MCN.P.504 difere de Hippidion que possui o metacônido e metastilido aproximadamente do mesmo tamanho e este último sem bordas angulares, as bordas labiais do protocônido e hipocônido são de formato seleneforme e o ectofléxido profundo, alcançando o linguafléxido (Ubilla \& Alberdi, 1990). Por outro lado, MCN.P.504 é similar a Equus (Amerhippus) por apresentar, em vista oclusal, o metacônido arredondado e o metastilido anguloso formando um "duplo laço", as bordas labiais do protocônido e hipocônido relativamente retilíneas, e o ectofléxido nunca alcançando o linguafléxido.

O MCN.P.504 é considerado de um adulto, pois apresenta raízes fechadas e todos os molares presentes, diferindo do espécime MNRJ 3784-V de Corumbá, Mato Grosso do Sul, descrito primeiramente por Souza-Cunha (1981) como Equus (Amerhippus) vandonii Souza-Cunha, 1981. Este espécime foi considerado como um juvenil de $E$. (A.) neogaeus por Alberdi et al. (2003), pois apresenta o pm2 sem desgaste e o m3 ainda não eclodido. Entretanto, MCN.P.504 é um adulto jovem, pois não apresenta um desgaste dentário tão acentuado como o do espécime MNRJ 3784-V de Equus (Amerhippus) neogaeus procedente de San Lorenzo, Argentina, descrito por Prado \& Alberdi (2008).

Comparando os dados morfométricos do espécime MCN.P.504 com os dados dos espécimes de Equus, figurados nos trabalhos de Alberdi et al. (2003, quadro 5), Rincón et al. (2006, quadro 1) e Prado \& Alberdi (2008, tabela 1) observase que o material aqui estudado se inclui dentro do intervalo de medidas apresentados por Equus (Amerhipus) neogaeus.

Do ponto de vista paleoambiental, a presença de Equus (Amerhipus) neogaeus sugere um ambiente de planícies abertas com vegetação xerofítica, com solos mais compactos e de clima mais seco e frio para esta localidade durante o Pleistoceno (Alberdi \& Prado, 1995).

$\mathrm{Na}$ região pampeana da Província de Buenos Aires, Argentina, são consideradas três idades que abrangem do Plioceno ao Pleistoceno: idade Ensenadense, Bonaerense e Lujanense (sensu Cione \& Tonni, 1995a; Cione et al., 2007). De acordo com Cione \& Tonni (1995b, 1999), Equus (Amerhipus) neogaeus aparece primeiramente no Pleistoceno superior, definindo uma biozona e caracterizando a idade Lujanense. Assim, a presença de E. (A.) neogaeus sugeriria uma idade Lujanense para o sítio do Paraná aqui descrito. Entretanto, pode não haver uma correlação exata de idades devido à posição geográfica do afloramento em relação aos afloramentos argentinos desta idade. Cartelle (1992) afirma que a fauna procedente de depósitos oriundos de cavernas e tanques das regiões Sudeste e Nordeste do Brasil (e.g. Lagoa Santa, MG; Vale do Ribeira, SP; Toca dos Ossos, BA) são sincrônicas e posteriores àquelas encontradas na Argentina durante o Lujanense, à exceção das faunas encontradas no Rio Grande do Sul. Por outro lado, idades obtidas através de datações absolutas $\left({ }^{230} \mathrm{Th} /{ }^{234} \mathrm{U}\right.$ em calcita recobrindo material fossilífero) sugerem que as deposições de restos de vertebrados pleistocênicos não são necessariamente resultados de eventos sincrônicos e sim de complexos processos que não guardam necessariamente ligação com os eventos paleoclimáticos (Auler et al., 2003).

Algumas datações de mamíferos pleistocênicos provenientes das cavernas de Lagoa Santa (e.g. Gruta Caianga e Gruta do Baú) apresentaram idades compreendidas entre 330.000 e 10.000 anos AP demonstrando uma mistura faunística, provavelmente resultado de diferentes causas e processos deposicionais (Auler et al., 2003). Entretanto, é tentativamente inferido que o espécime aqui estudado Equus (Amerhipus) neogaeus pertence à idade/piso Lujanense (Pleistoceno superior- Holoceno inferior). A datação absoluta bem como a identificação taxonômica mais precisa dos Megatheriinae e Gomphoteriidae associados, permitirão uma determinação mais segura da idade para esta assembleia fóssil.

A mastofauna pleistocênica do Estado do Paraná, na sua 


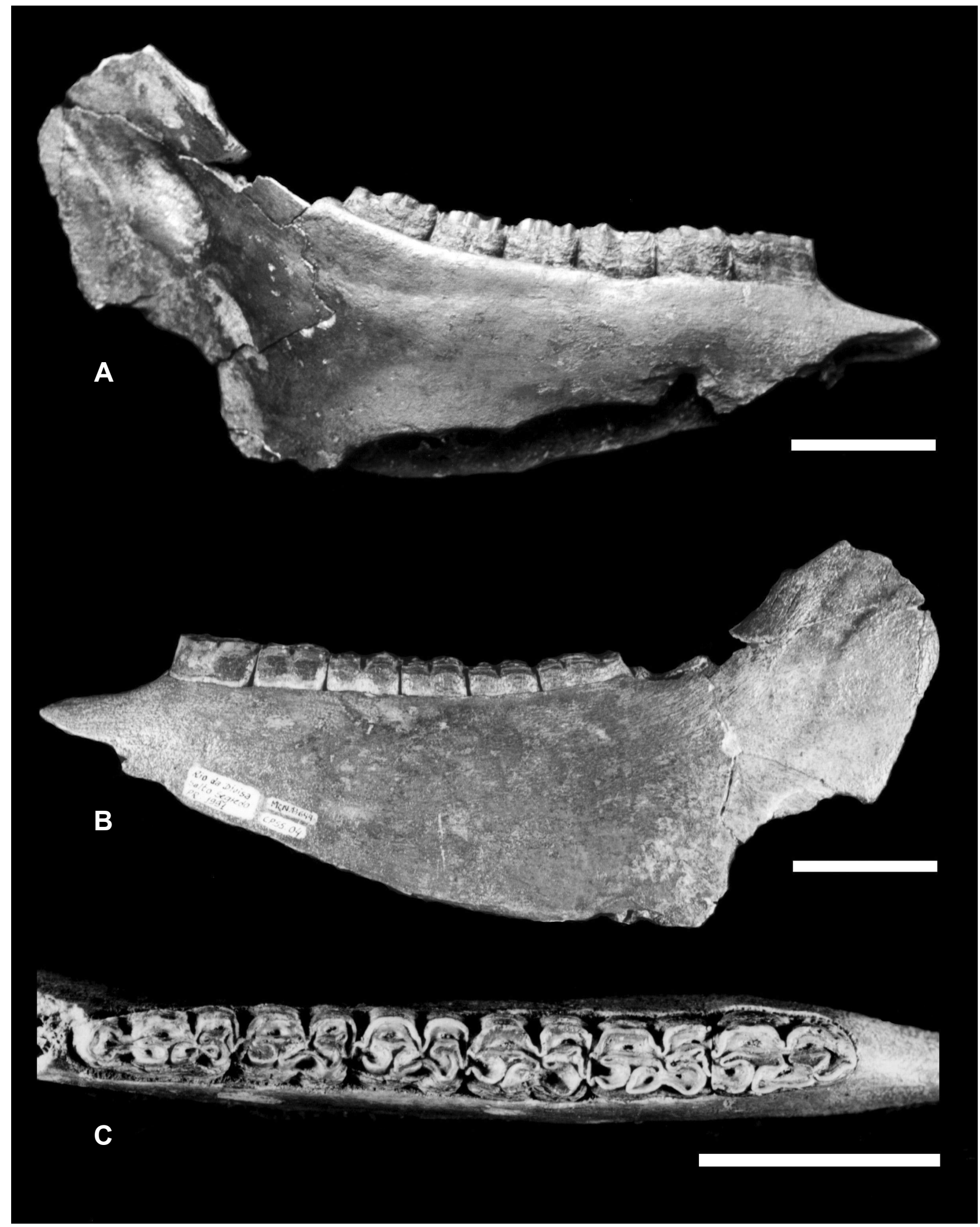

Figura 4. Equus (Amerhippus) neogaeus, MCN.P.504. A, dentário em vista medial; B, dentário em vista lateral; C, dentário em vista oclusal. Escalas $=5 \mathrm{~cm}$.

Figure 4. Equus (Amerhippus) neogaeus, MCN.P.504. A, dentary in medial view; B, dentary in lateral view; C, dentary in occlusal view. Scale bars $=5 \mathrm{~cm}$. 
Tabela 1. Dados morfométricos ( $\mathrm{mm}$ ) comparativos dos dentes inferiores do espécime MCN.P.504 com Equus (Amerhippus) segundo Eismann et al. (1988). Abreviaturas: $\mathbf{C}_{\text {sup }}$, comprimento superior; $\mathbf{L}_{\text {sup, }}$, Largura superior; $\mathbf{C}_{\text {base }}$, comprimento da base; $\mathbf{L}_{\text {base }}$, Largura da base; $\mathbf{H}$, altura; $\mathrm{C}_{\text {postf' }}$, comprimento da superfície mesiodistal do posterolofo.

Table 1. Comparative morphometric data $(\mathrm{mm})$ of the lower teeth of MCN.P.504 with Equus (Amerhippus) according to Eismann et al. (1988). Abbreviations: $\mathbf{C}_{\text {sup }}$, upper length; $\mathbf{L}_{\text {sup }}$, upper width; $\mathbf{C}_{\text {base }}$, base lenght; $\mathbf{L}_{\text {base }}$, base widht; $\mathbf{H}$, hight; $\mathbf{C}_{\text {postf' }}$ lenght of the mesiodistal surface of posteroloph.

\begin{tabular}{|c|c|c|c|c|c|c|c|}
\hline Dentes & Espécime & $\mathrm{C}_{\text {sup }}$ & $\mathrm{L}_{\text {sup }}$ & $\mathrm{C}_{\text {base }}$ & $\mathrm{L}_{\text {base }}$ & $\mathrm{H}$ & $\mathrm{C}_{\text {postr }}$ \\
\hline $\mathrm{P}_{2} \mathrm{i}$ & MCN.P 504 & 30,4 & 15,00 & & & & \\
\hline $\mathrm{P}_{3} \mathrm{i}$ & MCN.P 504 & 26,22 & 15,70 & & & & \\
\hline $\mathrm{P}_{4} \mathrm{i}$ & MCN.P 504 & 25,90 & 15,30 & & & & \\
\hline $\mathrm{M}_{1} \mathrm{i}$ & MCN.P 504 & 24,40 & 14,30 & & & & \\
\hline $\mathrm{M}_{2} \mathrm{i}$ & MCN.P 504 & 24,90 & 13,60 & & & & \\
\hline $\mathrm{M}_{3} \mathrm{i}$ & MCN.P 504 & 31,70 & 15,50 & & & & \\
\hline $\mathrm{DP}_{2} \mathrm{~d}$ & SGO.PV-23 & 43,8 & 24,8 & & & 28 & \\
\hline $\mathrm{P}_{3-4} \mathrm{i}$ & SGO.PV-23 & 27 & 16,5 & 24,3 & 15,5 & 51 & 8,5 \\
\hline $\mathrm{M}_{2} \mathrm{~d}$ & SGO.PV-23 & 26 & 15,5 & & & & 10,1 \\
\hline $\mathrm{M}_{3} \mathrm{~d}$ & SGO.PV-23 & 34 & 15,2 & & & & 9,9 \\
\hline $\mathrm{DP}_{3} \mathrm{~d}$ & V-3784 & 25,7 & 14 & & & & 10,5 \\
\hline $\mathrm{DP}_{4} \mathrm{~d}$ & V-3784 & 27 & 13,7 & & & & 9,9 \\
\hline $\mathrm{DP}_{3-4} \mathrm{i}$ & $\operatorname{sn}\left(\mathrm{M}_{2} ?\right)$ & 35 & 12 & & & 31 & 11,8 \\
\hline $\mathrm{P}_{2} \mathrm{~d}$ & MCN-6146 & 33,3 & 17 & 31,1 & 17,2 & 39,5 & 15,5 \\
\hline $\mathrm{P}_{2} \mathrm{i}$ & V-3627 & 31 & 13 & 28 & 12,3 & 32 & 13,8 \\
\hline $\mathrm{P}_{2} \mathrm{~d}$ & V-3784 & 30 & 13 & & & & \\
\hline $\mathrm{P}_{2} \mathrm{i}$ & MCN-6203 & 31,7 & 14,5 & & & 41 & 16,2 \\
\hline $\mathrm{P}_{2} \mathrm{i}$ & V-2830 & 33 & 15,2 & & & 62 & 16 \\
\hline $\mathrm{P}_{3} \mathrm{i}$ & V-2830 & 28 & 14,8 & & & 75 & 15 \\
\hline $\mathrm{P}_{3} \mathrm{i}$ & MCN-6203 & 28 & 17,1 & & & 49 & 15 \\
\hline $\mathrm{P}_{3-4} \mathrm{i}$ & V-2611 & 27 & 16 & 2 & 14 & 51 & 12,8 \\
\hline$P_{3-4} d$ & V-2811 & & & & & $>60$ & 13,6 \\
\hline$P_{3-4} d$ & MCN-6137d & 30,2 & 16,9 & 27 & 17 & 67 & 14 \\
\hline $\mathrm{P}_{3-4} \mathrm{i}$ & MCN-6136i & 30 & 17,5 & 27,2 & 15,5 & 73 & 14,5 \\
\hline$P_{3-4} d$ & MCN-6144d & 31 & 17,1 & 27 & 15,4 & 64 & 15 \\
\hline $\mathrm{P}_{3-4} \mathrm{i}$ & MCN-6142 & 30 & 16 & 27,5 & 15,9 & 68 & 15 \\
\hline$P_{3-4} d$ & MCN-6141 & 29,8 & 18 & 26,5 & 16 & 51 & 15 \\
\hline $\mathrm{P}_{3-4} \mathrm{i}$ & $\mathrm{MCN}-6145$ & 29 & 17,8 & 27,2 & 17,5 & 32 & 14 \\
\hline $\mathrm{P}_{3-4} \mathrm{i}$ & MCN-6149 & 27,7 & 17,7 & 26,1 & 14,7 & 44 & 13,4 \\
\hline $\mathrm{P}_{3-4}\left(\mathrm{M}_{1-2} ?\right) \mathrm{i}$ & V-2804 & 23,3 & 15 & & & 9 & \\
\hline $\mathrm{M}_{1} \mathrm{~d}$ & V-2468 & 24 & 14 & 23,7 & 14,5 & 33 & 9 \\
\hline $\mathrm{M}_{2} \mathrm{~d}$ & V-2468 & 23,5 & 15,5 & 22 & 16 & 35 & 7,8 \\
\hline $\mathrm{M}_{1-2} \mathrm{~d}$ & V-2808 & 26,3 & 14 & 24,5 & 12,6 & 67 & 11,9 \\
\hline $\mathrm{M}_{1-2} \mathrm{~d}$ & MCN-6139 & 26,5 & 15,6 & 24,5 & 15 & 69 & 15 \\
\hline $\mathrm{M}_{1-2} \mathrm{i}$ & MCN-6143 & 27 & 14 & 24,6 & 15,2 & 70 & 10,6 \\
\hline $\mathrm{M}_{1-2} \mathrm{i}$ & MCN-6135 & 29 & 15,5 & 24,7 & 14 & 68 & 11,4 \\
\hline $\mathrm{M}_{1-2} \mathrm{~d}$ & MCN-6138 & 25,2 & 16 & 23,7 & 15,3 & 58 & 11 \\
\hline $\mathrm{M}_{1-2} \mathrm{~d}$ & MCN-6148 & 26,7 & 16,3 & 25,3 & 15,5 & 33 & 10,8 \\
\hline $\mathrm{M}_{1-2} \mathrm{~d}$ & $\operatorname{sn}\left(\mathrm{M}_{2} ?\right)$ & 32 & 11,8 & 25 & 15 & 70 & 11,8 \\
\hline $\mathrm{M}_{1} \mathrm{~d}$ & V-3784 & 28 & 13,7 & & & & 10 \\
\hline $\mathrm{M}_{2} \mathrm{~d}$ & V-3784 & 29 & 11,7 & & & & 12 \\
\hline $\mathrm{M}_{3} \mathrm{i}$ & MCN-6179 & 29,7 & 13 & 33,1 & 12,5 & 68 & 7 \\
\hline $\mathrm{M}_{3} \mathrm{i}$ & MCN-6161 & 31 & 13 & 33 & 12,8 & 65 & 9,2 \\
\hline $\mathrm{M}_{3} \mathrm{~d}$ & MCN-6182 & 32,5 & 14 & 32,8 & 12,8 & 65 & \\
\hline $\mathrm{M}_{3} \mathrm{~d}$ & V-2812 & 28,3 & 12,6 & 30 & 12,2 & 59 & 8 \\
\hline $\mathrm{P}_{2} \mathrm{i}$ & MCPU-PV 137 & 30,4 & 15,00 & & & & \\
\hline $\mathrm{P}_{3} \mathrm{i}$ & MCPU-PV 137 & 26,22 & 15,70 & & & & \\
\hline $\mathrm{P}_{4} \mathrm{i}$ & MCPU-PV 137 & 25,90 & 15,30 & & & & \\
\hline $\mathrm{M}_{1} \mathrm{i}$ & MCPU-PV 137 & 24,40 & 14,30 & & & & \\
\hline $\mathrm{M}_{2} \mathrm{i}$ & MCPU-PV 137 & 24,90 & 13,60 & & & & \\
\hline $\mathrm{M}_{3} \mathrm{i}$ & MCPU-PV 137 & 31,70 & 15,50 & & & & \\
\hline
\end{tabular}


maioria, é constituída de materiais esparsos e fragmentários. Até o presente momento foram registrados os táxons Eremotherium laurillardi, Toxodon platensis, Stegomastodon waringi, Scelidodon sp. e Tapirus sp. (Stawiarski, 1944; Maack, 1947; Paulo Couto, 1975, 1978; Pillati \& Bortoli, 1978; Sedor et al., 2003), sendo que a ocorrência de Equus (Amerhipus) neogaeus vem aumentar o conhecimento sobre a diversidade de mamíferos extintos no Paraná, bem como no Brasil.

\section{AGRADECIMENTOS}

Os autores agradecem a COPEL, que forneceu irrestritamente a infraestrutura necessária para os trabalhos de campo e ao geólogo P. Levis pelo apoio nos trabalhos de campo, na ocasião da coleta. À Fundação Zoobotânica do Rio Grande do Sul por providenciar a estrutura para a realização desse trabalho. Ao saudoso biólogo R.R. Lange, que na ocasião notificou sobre a existência desse sítio fossilífero, L. Kerber por facilitar o acesso ao material comparativo, M.B. dos Santos pela revisão do texto e a R. I. Vezzosi pelo envio das fotos de material para comparação. À CAPES, pela concessão da bolsa de mestrado ao primeiro autor, e ao CNPq (processo 307940/2010-1) à A.M.R.

\section{REFERÊNCIAS}

Alberdi, M.T.; Cartelle, C. \& Prado, J.L. 2003. El registro Pleistoceno de Equus (Amerhippus) e Hippidion (Mammalia, Perissodactyla) de Brasil. Consideraciones paleoecológicas y biogeográficas. Ameghiniana, 40:173-196.

Alberdi, M.T. \& Frassinetti, D. 2000. Presencia de Hippidon y Equus (Amerhippus) (Mammalia, Perissodactyla) y su distribución en el Pleistoceno Superior de Chile. Estudios Geológicos, 56:279-290.

Alberdi, M.T. \& Prado, J.L. 1992. El registro de Hippidion Owen, 1869 y Equus (Amerhippus) Hoffstetter, 1950 (Mammalia, Perissodactyla) en América del Sur. Ameghiniana, 29:265-284.

Alberdi, M.T. \& Prado, J.L. 1993. Review of the genus Hippidion Owen, 1869 (Mammalia; Perissodactyla) from the Pleistocene of South America. Zoological Journal of the Linnean Society, 108:1-22.

Alberdi, M.T. \& Prado, J.L. 1995. Los équidos de América del Sur. In: M.T. Alberdi; G. Leone \& E.P. Tonni (eds.) Evolución biológica y climática de la región pampeana durante los últimos cinco millones de años. Museo Nacional de Ciencias Naturales CSIC, p. 293-308 (Monografía 12).

Alberdi, M.T. \& Prado, J.L. 2004. Los caballos fósiles de América del Sur. Una historia de 3 millones de años. Universidad Nacional del Centro de La Provincia de Buenos Aires, INQUAPA, 269 p. (Série Monográfica 3).

Auler, A.S.; Piló, L.B.; Smart, P.L.; Wang, X.; Neves, W.A. \& Edwards, R.L. 2003. Cronologia e processos de deposição da megafauna Quaternária em cavernas brasileiras: In: CONGRESSO DA ASSOCIAÇÃO BRASILEIRA DE ESTUDOS DO QUATERNÁRIO, 9, 2003. Anais, Recife, p. _.

Bergqvist, L.P.; Gomide, M.; Cartelle, C. \& Capilla, R. 1987. FaunasLocais de mamíferos pleistocênicos de Itapipoca/Ceará, Taperoá/ Paraíba e Campina Grande/Paraíba. Estudo Comparativo, biostratinômico e paleoambiental. Geociências, 2:23-32.

Boas, J.E.V. 1881. Om en fossil zebra-form fra brasiliensis Campos.
Med. et Tillaeg om to arter af salaegten Hippidion. Det kongelige Danske Videnskabernes Selskabs Skrifter naturvidenskabelig og mathematisk Afdeling, 1:305-330.

Carlini, A.A. \& Tonni, E.P. 2000. Mamíferos fósiles del Paraguay. Cooperación Técnica Paraguayo-Alemana, 108 p. (Proyecto Sistema Ambiental del Chaco/Región Oriental).

Cartelle, C.; Brant, W. \& Piló, L.B. 1989. A Gruta do Túnel de Santana (BA): morfogênese e paleontologia: In: CONGRESSO BRASILEIRO DE PALEONTOLOGIA, 11, 1989. Anais, Curitiba, p. 593-606.

Cartelle, C. 1992. Edentata e megamamiferos herbivoros extintos da Toca dos Ossos (Ourolândia, BA, Brasil). Programa de PósGraduação em Morfologia, Universidade Federal de Minas Gerais, Tese de Doutorado, 301 p.

Cione, L.A. \& Tonni, E.P. 1995a. Chronostratigraphy and "LandMammal Ages" in the Cenozoic of southern South America: principles, practices, and the "Uquian" Problem. Journal of Paleontology, 69:135-159.

Cione, A.L \& Tonni, E.P. 1995b. Bioestratigrafía y cronología del cenozoico superior de la región pampeana. In: M.T. Alberdi; G. Leone \& E.P. Tonni (eds.) Evolución climática y biológica de los últimos cinco millones de años. Museo Nacional de Ciencias Naturales, p. 47-74 (Monografias 12).

Cione, A.L \& Tonni, E.P. 1999. Biostratigraphy and chronological scale of uppermost Cenozoic in the Pampean area, Argentina. Quaternary of South America and Antarctic Peninsula, 12:23-51.

Cione, A.L.; Tonni, E.P.; Bargo, S.; Bond, M.; Candela, A.M.; Carlini, A.A.; Deschamps, C.M.; Dozo, M.T.; Esteban, G.; Goin, F.J.; Montalvo, C.I.; Nasif, N.; Noriega, J.I.; Ortiz-Jaureguizar, E.; Pascual, R.; Prado, J.L.; Reguero, M.A.; Scillato-Yané, G.J.; Soibelzon, L.; Verzi, D.H.; Vieytes, E.C.; Vizcaíno, S.F. \& Vucetich, M. G. 2007. Mamíferos continentales del Mioceno tardío a la actualidad en la Argentina: cincuenta años de estúdios. Ameghiniana, 11:257-278.

Dias Neto, C.M.; Born, P.A. \& Chahud, A. 2008. Ocorrência de mamíferos extintos do Pleistoceno na localidade de Lagoa da Pedra, Município de Igaci, Alagoas. Revista Universidade Guarulhos, Geociencias, 7:110-115.

Eisenmann, V.; Alberdi, M.T.; De Giuli, C. \& Staesche, V. 1988. Collected papers after the "New York Internacional Hipparion Conference, 1981". In: M. Woodburne \& P. Sondaar (eds.) Studying fossil horses, 1, Methodology. E. J. Brill, p. 1-72.

Ficcarelli, G.; Coltorti, M.; Moreno Espinosa, M.; Pieruccini, P.L.; Rook, L. \& Torre, D. 2003. A model for the Holocene extinction of the mammals megafauna in Ecuador. Journal of South American Earth Sciences, 15:835-845.

Ghilardi, A.M.; Fernandes, M.A. \& Bichuette, M.E. 2011. Megafauna from the Late Pleistocene-Holocene deposits of the Upper Ribeira karst area, southeast Brazil. Quaternary International, 245:369-378. doi:10.1016/j.quaint.2011.04.018

Guérin, C. 1991. La faune de vertébrés du Pléistocéne supérieur de l'aire archéologique de Sao Raimundo Nonato (Piaui, Brésil). Comptes Rendus de l'Académie des Sciences, 312:567-572.

Lund, P.W. 1840. Nouvelles recherches sur la faune fossile du Brésil. Annales Science Naturalles, 13:310-319.

Lund, P.W. 1842. Fjerde Afhandling: Fortsaettelse af Pattedyrene. Det köngelige Danske Videnskabernes Selskabs Naturvidenskabelige og Mathematiske Afhandlinger, 9:137-208.

Lund, P.W. 1846. Meddlelse af det Udbytte de I 1844 undersögte Knoglehuler Have avgivet til hundskaben om Brasiliens Dyreverden för sidste Jordomvaeltning. Det köngelige Danske Videnskabernes Selskabs Naturvidenskabelige og Mathematiske Afhandlinger, 12:57-94. 
Kerber, L. \& Oliveira, E.V. 2008a. A presença de Equus (Perissodactyla, Equidae) e Neuryurus (Cingulata, Glyptodontidae) no Quaternário do Município de Quaraí, oeste do Rio Grande do Sul. Cadernos de Pesquisa, Série Biológica, 20:18-25.

Kerber, L. \& Oliveira, E.V. 2008b. Fósseis de vertebrados da Formação Touro Passo (Pleistoceno Superior), Rio Grande do Sul, Brasil: atualização dos dados e novas contribuições. Gaea, 4:49-64. doi:10.4013/gaea.20082.02

Maak, R. 1947. Breves notícias sobre a geologia dos estados do Paraná e Santa Catarina. Arquivos de Biologia e Tecnologia, 2:141-150

MacFadden, B.J. 1997. Pleistocene horses from Tarija, Bolívia, and vality of genus Onohippidium (Mammalia: Equidae). Journal of Vertebrate Paleontology, 17:199-218.

MacFadden, B.J.; Siles, O.; Zeitler, P.; Johnson, N.M. \& Campbell, K.E. 1983. Magnetic polarity stratigraphy of the Middle Pleistocene (Ensenadan) Tarija Formation of southern Bolivia. Quaternary Research, 19:172-187.

Martinez, J.N. 2004. San Sebastían: un nuevo registro de mamíferos del Pleistoceno superior en Piura. Comentarios sobre los mamíferos continentales del Pleistoceno de la Región PiuraTumbes. In: CONGRESO PERUANO DE GEOLOGIA, 12, 2004. Actas, Lima, p. 485-488.

Marshall L.G.; Butler, R.F.; Drake, R.E. \& Curtis, G.H. 1982. Geochronology of type Uquian (Late Cenozoic) Land Mammal Age, Argentina. Science, 216:986-989.

Oliveira, E.V. 1992. Mamíferos fósseis do Quaternário do Estado do Rio Grande do Sul, Brasil. Programa de Pós-Graduação em Geociências, Universidade Federal do Rio Grande do Sul, Dissertação de Mestrado, 118 p.

Paula Couto, C. 1944. Sobre a ocorrência dos gêneros Hippidion e Toxodon Owen, no Pleistoceno do Rio Grande do Sul. Boletim do Museu Nacional, Nova Série Geologia, 2:1-12.

Paula Couto, C. 1975. Mamíferos do Quaternário do Sudeste Brasileiro. Boletim Paranaense de Geociências, 33:89-132.

Paula Couto, C. 1978. Presença de Toxodon platensis Owen no Pleistoceno do Paraná. Inheringia, Série Geológica, 5:55-59.

Pillati, F. \& Bortoli, C. 1978. Presença de Haplamastodon, um Mastodonte Quaternário do Estado do Paraná. Acta Geológica Leopoldensia, 3:3-13.

Porpino, K.O.; Santos, M.F.C.F. \& Bergqvist, L.P. 2004. Registros de mamíferos fósseis no Lajedo de Soledade, Apodi, Rio Grande do Norte, Brasil. Revista Brasileira de Paleontologia, 7:349-358.

Prado, J.L. \& Alberdi, M.T. 1994. A quantitative review of the horse Equus from South America. Palaeontology, 37:459-481.

Prado, J.L.; Alberdi, M.T., \& Reguero, M.A. 1998. El registro más antiguo de Hippidion Owen, 1869 (Mammalia, Perissodactyla) en América del Sur. Estúdios Geológicos, 54:85-91.

Prado, J.L. \& Alberdi, M.T. 2008. Restos de Hippidion y Equus (Amerhippus) procedentes de las barrancas de San Lorenzo, Pleistoceno tardío (Provincia de Santa Fé, Argentina). Revista Española de Paleontología, 23:225-236.

Price, L.I. 1944. O depósito de vertebrados pleistocênicos de Águas de Araxá (Minas Gerais). Anais da Academia de Ciências, 16:193-195.

Reguero, R.; Candela, A.M. \& Alonso, R. 2007. Biochronology and biostratigraphy of Uquía Formation (Pliocene-early Pleistocene, NW Argentina) and its significance in the Great American Biotic Interchange. Journal of South American Earth Sciences, 23:1-16. doi:10.1016/j.jsames.2006.09.005

Ribeiro, A.M.; Sedor, F.A. \& Silva, D.D. 2005. Equus (Amerhippus) neogaeus (Lund, 1840) (Equidae, Perissodactyla, Mammalia) no Quaternário do Estado do Paraná, Brasil. Revista Ciência e Natura. Edição Especial, p.72.

Ribeiro, A.M. \& Scherer, C.S. 2009. Mamíferos do Pleistoceno do Rio Grande do Sul. In: A.M. Ribeiro; S.G. Bauermann \& C.S. Scherer (orgs.) Quaternário do Rio Grande do Sul - integrando conhecimentos. p. 171-191 (Monografias da Sociedade Brasileira de Paleontologia 1).

Rincón, A.D.; Alberdi, M.T. \& Prado, J.L. 2006. Nuevo registro de Equus (Amerhippus) santaeelenae (Mammalia, Perissodactyla) del pozo de asfalto de Inciarte (Pleistoceno Superior), estado Zulia, Venezuela. Ameghiniana, 43:529-538.

Rolim, J.L. 1971. Sobre alguns Mamíferos fósseis de Lagoa da Pedra - Munícipio de Santa Cruz do Capibaribe - Pernambuco. Estudos e Pesquisas, série B, 3:1-19.

Salas, R.; Urbina, M \& DeVries, T. 2004. Los mamíferos de Quebrada El Jahuary (Arequipa, Peru): fauna típica del Pleistoceno tardio de la costa peruana. In: CONGRESO PERUANO DE GEOLOGIA, 12, 2004. Actas, Lima, p. 525-528.

Salles, L. O.; Cartelle, C.; Guedes, P.G.; Boggiani, P.C.; Janoo, A. \& Russo, C.A.M. 2006. Quaternary Mammals from Serra da Bodoquena, Mato Grosso do Sul, Brazil. Boletim do Museu Nacional. Zoologia, 521:1-12.

Sánchez, B.; Prado, J.L. \& Alberdi, M.T. 2006. Ancient feeding, ecology and extinction of Pleistocene horses from the Pampean region (Argentina). Ameghiniana, 43:427-436.

Scherer, C.S. \& Da Rosa, A.A.S. 2003. Um equídeo fóssil do Pleistoceno de Alegrete, RS, Brasil. Pesquisas em Geociências, 30:33-38.

Silva, F.M.; Filgueiras, C.F.C.; Barreto; A.M.F. \& Oliveira, E.V. 2010. Mamíferos do Pleistoceno Superior de Afrânio, Pernambuco, nordeste do Brasil. Quaternary and Environmental Geosciences, 2:1-11.

Souza Cunha, F.L. 1959. Mamíferos fósseis do Pleistoceno do Rio Grande do Sul. I - Ungulados. Departamento Nacional de Produção Mineral, Divisão de Geologia e Mineralogia, 68 p. (Boletim 202).

Souza Cunha, F.L. 1981. Equus (Amerhippus) vandonii n. sp. um novo cavalo fóssil de Corumbá, Mato Grosso do Sul, Brasil. Boletim do Museu Nacional (Geologia), 40:1-19.

Stawiarski, V. 1944. Em busca de um fóssil. Revista do Museu Nacional, 2:17-24.

Ubilla, M. \& Alberdi, M.T. 1990. Hippidion sp. (Mammalia, Perissodactyla, Equidae) en sedimentos del Pleistoceno Superior del Uruguay (edad mamifero Lujanense). Estudios Geológicos, 46:453-464.

Vidal, N. 1946. Contribuição ao conhecimento da paleontologia do nordeste brasileiro. Notícias sôbre a descoberta de vertebrados pleistocênicos no Município de Pesqueira, em Pernambuco. Boletim do Museu Nacional, (nova série, Geologia), 6:1-19.

Webb, S.D. 1991. Ecogeography and the Great American interchange. Paleobiology, 17:266-280.

Winge, H. 1906. Jordgundne og nulevende Hovdyr (Ungulata) fra Lagoa Santa, Minas Gerais, Brasilien. Med udsigt over gumlernes indbyrdes slaegtskab, E. Museo Lundii, 3:1-239.

Received in February, 2012; accepted in August, 2012. 